\title{
EEG CONNECTIVITY - INFORMED COOPERATIVE ADAPTIVE LINE ENHANCER FOR RECOGNITION OF BRAIN STATE
}

\author{
Saeid Sanei ${ }^{*}$, Clive Cheong Took ${ }^{\dagger}$, Delaram Jarchi $^{\ddagger}$, and Ales Prochazka**† \\ ${ }^{*}$ School of Science and Technology, Nottingham Trent University, UK \\ ${ }^{\dagger}$ Department of Electrical Engineering, Royal Holloway University of London, UK \\ ${ }^{\ddagger}$ School of Computer Science and Electrical Engineering, University of Essex, UK \\ ** Department of Computing and Control Engineering, University of Chemistry and Technology, Czech Republic \\ ${ }^{\dagger \dagger}$ Department of Neurology, Charles University, Faculty of Medicine in Hradec Kralove, Czech Republic
}

\begin{abstract}
Bursts of sleep spindles and paroxysmal fast brain activity waveforms have frequency overlap whilst generally, paroxysmal waveforms have shorter duration than spindles. Both resemble bursts of normal alpha activity during short rests while awake with closed eyes. In this paper, it is shown that for a proposed cooperative adaptive line enhancer, which can both detect and separate such periodic bursts, the combination weights are consistently different from each other. The outcome suggests that for accurate modelling of the brain neuro-generators, the brain connectivity has to be precisely estimated and plugged into the adaptation process.
\end{abstract}

Index Terms - Cooperative adaptive line enhancer, EEG, brain connectivity, alpha wave, paroxysmal, sleep spindles.

\section{INTRODUCTION}

The state of brain activity is recognised up to a high extent by temporal and spatial characteristics of the electroencephalography (EEG) waveforms and the neuronal communications between the brain zones [1]. As a normal brain activity, sleep spindles are peculiar, random in time, oscillatory brain patterns associated with the second and third stages of sleep. They last between 0.5 to over one second, and have frequencies in alpha range to early beta range (i.e. 10-15 Hz). Their visual scoring is cumbersome and user dependent. On the other hand, as an abnormal brain activity, in tonc-clonic seizure patients, the brain often generates bursts of paroxysmal periodic polyspikes, which are fast and short bursts of brain activity with frequencies around lower beta range, appearing across the left posterior brain quadrant with a spatial distribution which is neither fully generalised nor truly focal. The spindles often appear in all the EEG channels but mostly appear around the mid-line of the brain on both centro-parietal (for early spindles) and frontal (for late spindles) zones of the brain. Both waveforms are also very similar to frontal-central beta activity as well as normal alpha activity during short resting periods while awake.
Detection of such waveforms and their separation from normal brain bursts are necessary for recognition and scoring either the sleep stages or the severity of tonic-clonic seizure and distinguishing them from normal alpha and beta bursts. In addition, the spatial distinction of these two waveforms and the way they evolve within the brain indicate their differences and set the criteria for their detection and characterisation.

Efforts have been made to detect some of these waveforms (e.g., spindles) automatically [2]. These methods are either based on time-frequency [3] [4], source separation [5], or other approaches [2]. From Figure 1 the similarity between these activities is visible. Without clinical examination of the patient, it is easy to make mistake between them particularly if these waveforms are elicited around the same time. One example is when the paroxysmal seizure happens during sleep.

\section{METHODOLOGY}

As for the data, the sleep EEG signals are preprocessed using the method in [6] to mitigate the effect of eye-blinks before applying our proposed spindle detection method. The data are processed block by block of $0.5 \mathrm{sec}$ each and the sampling rate is 256 samples/sec.

\subsection{Cooperative Learning}

A distributed adaptive network is a collection of connected agents that have some learning abilities enabling them to sense and adapt to the streaming data. Moreover, these networks are able to collaborate and share their information on a local level. This collaboration results in the complex behaviour of the network and diffusion of information over the entire system. The performance of these self-organized networks depends on the learning abilities and localized cooperation of the inter-connected nodes of the network [7]. After all, the objective of the network is to discover some global information and resolve a global task by using the distributed information of all the agents. Figure 1 shows an 

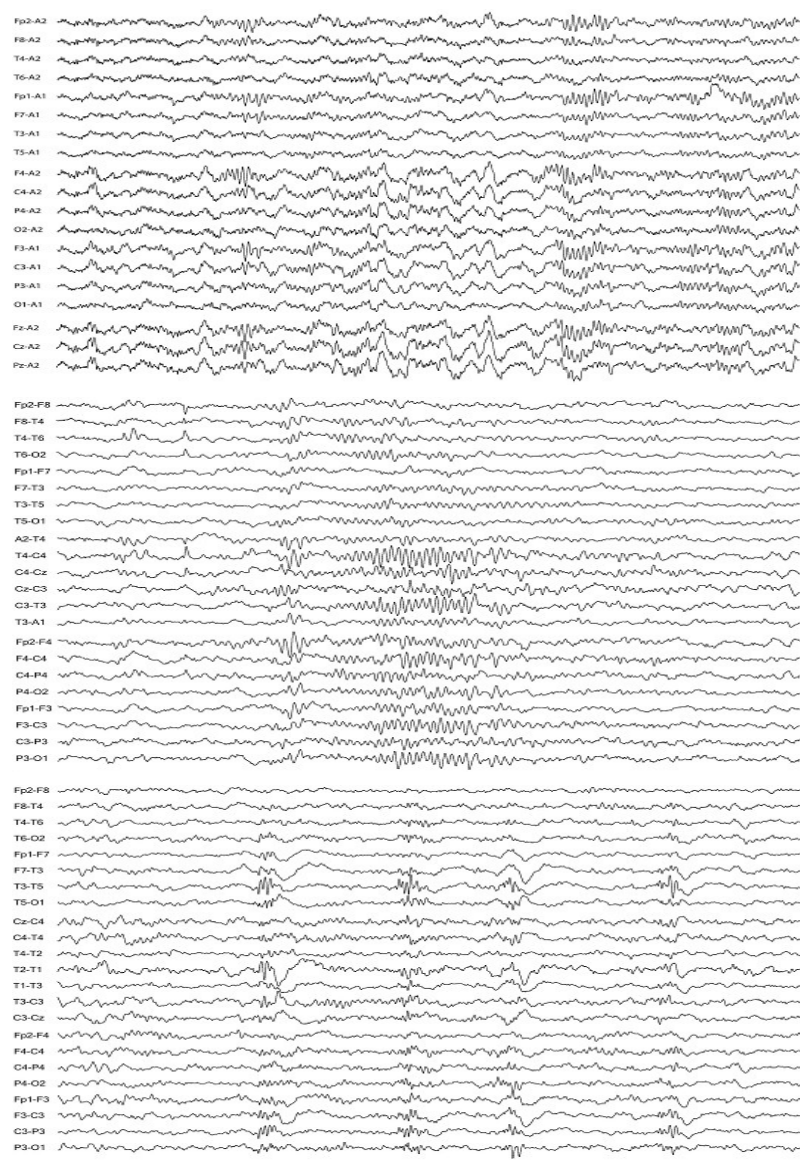

Fig. 1. EEG with bursts of beta activity (top), sleep EEG with bursts of spindles (middle), and seizure EEG with bursts of paroxysmal activity (bottom).

example of such a network comprising $N$ agents,where each agent $k$ is connected to some neighbours in a set represented by $N_{k}$ that can communicate with.

Consider $N_{k}$ as the neighbours of node $k$, including $k$ itself. To model the problem, we consider a connected network consisting of $N$ nodes. Such a network can be seen in Figure 2. Each node attempts to estimate a $1 \times \mathrm{M}$ unknown vector $\mathbf{w}_{k}^{o}$ from the collected measurements. Each node $k$ of the network has access to scalar measurements $d_{k}$ as its target, and an $1 \times \mathrm{M}$ regression vector $x_{k}(i)$ at each time instant $i \geq$ 0 . The information at each node is assumed to be related to the $\mathbf{w}_{k}^{o}$ via a linear regression model:

$$
d_{k}(i)=x_{k}^{T}(i) \mathbf{w}_{k}^{o}+n_{k}(i)
$$

where $n_{k}(i)$ is the measurement noise at node $k$ in time instant $i$ and $T$ refers to transpose of a vector. In the context of diffusion adaptation (DA), this leads to an optimization problem which minimizes a cost function, $J_{k}(\mathbf{w})$, for each node $k$ as shown in Figure 2, proportional to the difference between an estimate and the corresponding objective, resulting

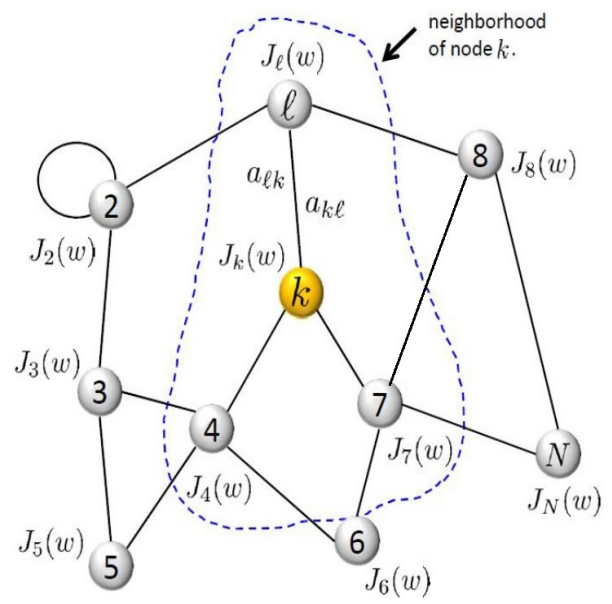

Fig. 2. A cooperative network and the neighbourhood of node $k$.

in the following two-step solution which involves adaptation and combination respectively:

$$
\begin{gathered}
\phi_{k}(i)=\mathbf{w}_{k}(i-1)+\mu_{k} d_{k} x_{k}^{T}(i) \mathbf{w}_{k}(i-1) x_{k}(i) \\
\mathbf{w}_{k}(i)=\sum_{l \in N_{k}} a_{l k} \phi_{l}(i)
\end{gathered}
$$

where $\mu_{k}>0$ is the step-size parameter used by node $k, \mathbf{w}_{k}$ denotes the estimate of actual filter parameters, and $a_{l k}$ are nonnegative combination weights which satisfy

$$
\sum_{l \in N_{k}} a_{l k}=1, \quad \text { and } \quad a_{l k}=0 \quad \text { if } \quad l \notin N_{k}
$$

The adaptation and combination steps can be performed in any order. Generally, there are no clues about the values of $a_{l k}$ and often for an $N$ node neighbourhood each link weight is considered equal to $1 / N$ [7]. Single- and multi-task cooperative networks have diverse range of applications such as in [8] [9] [10]. In application to EEG, accurate estimation of the combination parameters becomes very crucial as they play the major role in both recognition of brain state and classification of the states eliciting similar EEG waveforms. The estimation of brain connectivity (often proportional to Granger causality measure) can be achieved using different methods.

\subsection{Cooperative Adaptive Line Enhancer}

Adaptive line enhancer (ALE) was introduced by Widrow [11] and widely used for separation of sinusoid or narrowband periodic signals from their mixtures with broad-band noise. The general block diagram of a traditional single channel ALE is depicted in Figure 3. The ALE input $s(t)$ is assumed to be the sum of a narrow-band signal $x(t)$ and a broad-band signal $n(t)$. The parameters of the filter, w, 


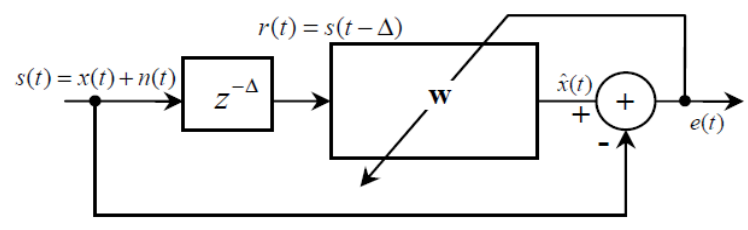

Fig. 3. Conventional ALE.

are recursively adapted and estimated in such a way that the statistical mean squared error (MSE), $E\left[e^{2}(t)\right]$, where $E[$. stands for statistical expectation, is minimized. The ALE operates by virtue of the difference between the correlation lengths of $x(t)$ and $n(t)$. The delay parameter $\Delta$ should be chosen larger than the correlation length of $n(t)$, but smaller than the correlation length of $x(t)$. The correlation length is equal to the longest interval between the two correlated samples of the signal, i.e., where the signal autocorrelation falls to zero. In this case, it is possible for $\mathbf{w}$ to make a $\Delta$-step ahead prediction of $x(t-\Delta)$ based on the present and past samples of $s(t-\Delta)$. However, $\mathbf{w}$ is not able to predict $n(t)$ from the knowledge about present and past samples of $n(t-\Delta)$. As a result, after the convergence of $\mathbf{w}$ to its optimal values, the error signal $e(t)$ is approximately equal to $n(t)$ and the ALE output $x(t)$ is approximately equal to $x(t)$. New ALEs have been proposed with particular applications to wideband periodic signals buried in non-Gaussian noise [12] or three-dimensional periodic signals [6].

ALE has applications in many areas such as communications, sound, and biomedical signal processing. It operates on a single channel and assumes that the periodic signal exists throughout the data segment. Therefore, in places where a burst of periodic signal is propagated over a connected network the method is not applicable. Here, we exploit the fact that EEG is generally a multichannel data and the normal or abnormal EEG source locations are neither known in advance nor steady in any certain brain zone. These include the sources of short bursts of normal alpha and beta activities, sleep spindles and paroxysmal activity. However, normal alpha often elicits from posterior brain zone (or from midlateral zone if it is a mu rhythm) and beta activity from frontal zone. On the other hand, it has been well established that, during sleep there is no alpha- or beta-range activities.

Nevertheless, the main hypothesis in this work is that, during the above different brain states/activities, the connectivity patterns between various brain zones vary considerably. Hence, the brain connectivity parameters can be good estimates of the combination weights for a cooperative ALE (CoALE) introduced in [13] to best estimate the desired waveform and classify the above waveforms. For a CoALE the target signal in equation (2) is replaced with the shifted input signal, i.e.,

$$
\phi_{k}(i)=\mathbf{w}_{k}(i-1)+\mu_{k} x_{k}(i-\Delta) x_{k}^{T}(i) \mathbf{w}_{k}(i-1) x_{k}(i)
$$

However, the combination equation follows equation (3).

\subsection{Brain Connectivity}

Generally, the brain connectivity measures follow the Granger causality principle. In order to estimate the combination weights in each time interval for more accurate estimation of cooperative filter parameters, $\mathbf{x}_{k}$ the Stockwell timefrequency transform (S-transform) [14] is employed here. This method is more accurate and less sensitive to the changes in time-frequency parameters compared to the autoregressive based methods [15]. It is defined as:

$$
\mathbf{x}_{k}(\tau, f)=\int_{-\infty}^{\infty} \mathbf{x}_{k}(t) \frac{|f|}{\sqrt{2 \pi}} e^{-\frac{(\tau-t)^{2} f^{2}}{2}} e^{-i 2 \pi f t}
$$

Then, the cross-spectrum (or S-coherency) of the signal is defined as:

$$
C_{k l}^{(S T)}(t, f)=\frac{S_{k l}^{(S T)}(t, f)}{\sqrt{S_{k k}^{(S T)}(t, f) S_{l l}^{(S T)}(t, f)}}
$$

where

$$
S_{k l}^{(S T)}(t, f)=\left\langle\mathbf{X}_{k}(t, f) \mathbf{X}_{k}^{*}(t, f)\right\rangle
$$

Obviously, $\mathbf{X}_{k}(t, f)$ is complex-valued. The imaginary part of S-coherency (ImSCoh) is related to the phase difference between the signals $x_{k}(t)$ and $x_{l}(t)$ at each frequency $f$; e.g., if $\operatorname{ImSCoh}$ is positive then, $x_{k}(t)$ and $x_{l}(t)$ are interacting and $x_{k}(t)$ leads $x_{l}(t)$. The cross-spectra values are then used in estimation of the combination weights as [16]:

$$
a_{k l}^{(S T)}=\frac{\max \left(\operatorname{Im}\left(C_{k l}^{(S T)}(t, f)\right), 0\right)}{\sum_{l \in N_{k}} \max \left(\operatorname{Im}\left(C_{k l}^{(S T)}(t, f)\right), 0\right)}
$$

The estimated $a_{k l}$ are then plugged into the adaptation equation for estimation of the DA weights $\mathbf{w}_{k l}$. The application of S-coherency in connection with cooperative learning has been reported for classification of hand clockwise and anticlockwise movements [17] and for EEG-based tremor detection in patients suffering from Parkinson's disease [16].

\section{DETECTION OF BURSTS OF EEG PERIODIC WAVEFORMS}

In the data used here, the spindle, paroxysmal wave, short alpha, and short beta last between 0.5 to 1.5 seconds. All the waveforms have approximately constant (but different) amplitudes. However, the amplitudes are modulated by the EEG 


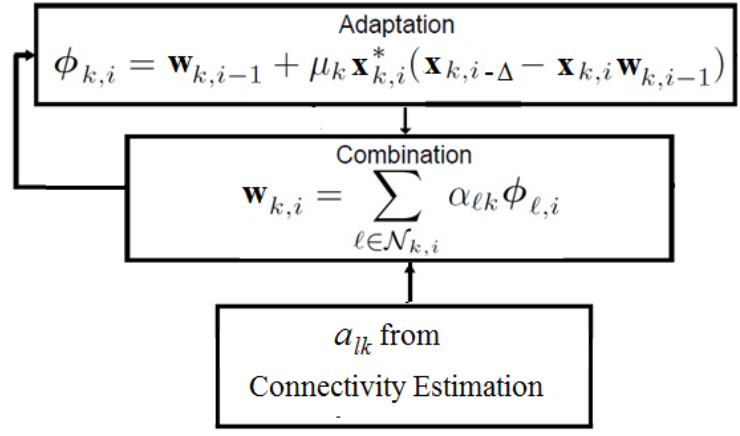

Fig. 4. EEG connectivity informed CoALE.

variation and also distributed over the neighbouring electrodes. This is seen in Figure 1 very clearly. From this figure, the waveforms are seen to be propagated to the neighbouring channels. The amount of propagation is highly dependent on the cooperation levels between various brain zones as estimated by the brain connectivity and reflected in the combination coefficients. Here, the sets of EEG signals are converted to referential mode ( $w . r . t$ mid-zone electrode, $C_{z}$,) and preprocessed using the method in [6] to eliminate the effects of eye-blinks on the signals. Then, non-overlapping consecutive 500 msec (125 samples) segments were processed by the proposed informed CoALE. The delay, $\Delta$, was selected as $4 / f_{0}$, where $f_{0}$ is a peak frequency of a labelled periodic burst in any of the above related EEG segments.

After designing the filter of Figure 4 for restoration of the rhythmic waveforms, it is applied to the multichannel EEGs and the results compared with those of [3] and [4] as well as the one scored by expert clinicians as the gold standard. The results of applying the CoALE are shown in Table 1. In this table we also present the outcome for CoALE where the combination weights for each agent are similar and therefore, the parameter vector $\mathbf{w}_{k}$ is only the average of the aggregate vector $\phi_{k l}$ over $N$, the number of agents in the neighbourhood. Although for all the three methods the spindles are detected correctly, estimation of their exact durations involves error. This error, $\epsilon$, is calculated as follows:

$$
\epsilon=1-\frac{\text { actual and estimated signals overlap duration }}{\text { duration of actual signal }}
$$

From Table 1 it is clear that, although single channel based methods are not always able to detect the full duration of burst waveforms, these particular methods have shown to be very successful in detection of the EEG sleep spindles. On the other hand, if the traditional DA-based cooperative filtering is used in the ALE design, without any prior information about the cooperation weights which quantify the brain connectivity, the system is not sufficiently effective. In the proposed method the cooperative weights are estimated for each
Table 1. The error in detection of full duration of each spindle using the proposed (multichannel) informed CoALE, singlechannel based methods, and the CoALE where the cooperative coefficients are considered equal for each agent $\mathrm{k}$ and its neighbourhood.

\begin{tabular}{|c|c|c|c|}
\hline Eethod & $\begin{array}{c}\text { Beta } \\
\text { activity }\end{array}$ & Spindle & $\begin{array}{c}\text { Paroxysmal } \\
\text { activity }\end{array}$ \\
\hline Time-frequency [3] & $24 \%$ & $26 \%$ & $27 \%$ \\
Tensor-based [4] & $16 \%$ & $17 \%$ & $17 \%$ \\
$\begin{array}{c}\text { Non-Cooperative ALE } \\
\text { (independent channels) }\end{array}$ & $20 \%$ & $19 \%$ (ave.) & $19 \%$ \\
$\begin{array}{c}\text { CoALE (all } a_{k l} \text { the same, i.e. } \\
\text { their average for each } k \text { ) } \\
\text { Proposed Informed CoALE }\end{array}$ & $8.5 \%$ & $8.5 \%$ & $7.5 \%$ \\
\hline
\end{tabular}

Table 2. Classifications accuracy for discriminating between each two brain states using (a) connectivity parameters and support vector machines (SVM) classification and (b) connectivity-informed CoALE.

\begin{tabular}{|c|c|c|}
\hline Two brain waveforms compared & $\begin{array}{c}\text { Connectivity } \\
\text { and SVM }\end{array}$ & $\begin{array}{c}\text { Connectivity- } \\
\text { inf. CoALE }\end{array}$ \\
\hline alpha and beta bursts & $92 \%$ & $100 \%$ \\
alpha burst and sleep spindle & $90 \%$ & $98.2 \%$ \\
sleep spindle and seizure paroxysmal & $91.6 \%$ & $96.6 \%$ \\
sleep spindle and beta burst & $88 \%$ & $100 \%$ \\
alpha burst and seizure paroxysmal & $90.6 \%$ & $100 \%$ \\
beta burst and seizure paroxysmal & $87.8 \%$ & $97.4 \%$ \\
sleep spindle and beta burst & $92.5 \%$ & $100 \%$ \\
\hline
\end{tabular}

500msec segment and included in the combination and adaptation processes. Therefore, the method can not only detect the bursts of periodic waveforms correctly but also their full durations are precisely detected and labelled.

In Table 2 the classifications accuracy for discriminating between each two brain states are depicted. 25 segments for each case (short alpha burst, short beta burst, spindle, and seizure paroxysmal activity) have been used.

\section{CONCLUSIONS}

Unlike in conventional diffusion adaptation applications where the weights are often approximated (or considered equal), thanks to the brain connectivity measures, in the case of brain research, these parameters are estimated for each epoch of the data with reasonable accuracy. A good example explored here, is the differentiation between different short bursts of rhythmic brain activities each representing a different state of the brain. Sleep spindles, paroxysmal seizure activity, and transient alpha or beta waves are detected and classified using the proposed algorithm. The method may be extended to differentiate many brain rhythmic activities which have different spatial distributions and even to other signals recorded using body sensor networks [18].

\section{ACKNOWLEDGEMENT}

We acknowledge FN HK 00179906 and project PERSONMED, Reg. CZ.02.1.010.00.017-0480007441, CZ, funds. 


\section{REFERENCES}

[1] S. Sanei, Adaptive Processing of Brain Signals, John Wiley \& Sons, 2013.

[2] J. Zygierewicz, K. J. Blinowska, P. J. Durka, W. S. S. Niemcewicz, and Androsiuk W., "High resolution study of sleep spindles," IEEE Signal Processing Letters, vol. 12, no. 10, pp. 721-724, 2006.

[3] S. Mahvash Mohammadi, M. Ghavami, S. Kouchaki, and S. Sanei, "Improving time-frequency domain sleep eeg classification via singular spectrum analysis," Journal of Neuroscience Methods, vol. 273, pp. 96-106, 2016.

[4] S. Kouchaki, S. Sanei, E. Arbon, and D.-J. Dijk, "Tensor based singular spectrum analysis for automatic scoring of sleep eeg," IEEE Transactions on Neural Systems and Rehabilitation Engineering, vol. 23, no. 1, pp. 1-9, 2015 .

[5] E. M. Ventouras, P. Y. Ktonas, H. Tsekou, T. Paparrigopoulos, I. Kalatzis, and C. R. Soldatos, "Independent component analysis for source localization of EEG sleep spindle components," Computational Intelligence and Neuroscience, vol. 1, pp. 329-436, 2010.

[6] L. Shoker, S. Sanei, and J. Chambers, "Artifact removal from electroencephalograms using a hybrid bss-svm algorithm," IEEE Signal Processing Letters, vol. 12, no. 10, pp. 721-724, 2006.

[7] A. Sayed, "Adaptation, learning, and optimization over networks," Foundations and Trends in Machine Learning, vol. 7, no. 4-5, pp. 311-801, 2014.

[8] S. Monajemi, S. Sanei, and S. H. Ong, "Information reliability in complex multitask networks," Future Generation Computer Systems, vol. 83, pp. 485-495, 2018.

[9] S. Monajemi, S. Sanei, and S. H. Ong, "Dictionary learning applications for hep-2 cell classification," Signal Processing and Machine Learning for Biomedical Big Data, Book Chapter, pp. 369-380, 2018.

[10] A. Latifi, A. Rastegarnia, A. Khalili, and S. Sanei, "Agent-based decentralized optimal charging strategy for plug-in electric vehicles," IEEE Transactions on Industrial Electronics, vol. 66, no. 5, pp. 3668-3680, 2019.
[11] B. Widrow, "Adaptive noise cancelling: Principles and applications," Proceedings of IEEE, vol. 63, no. 12, pp. 1692-1716, 1975.

[12] S. Sanei, T. K. M. Lee, and V. Abolghasemi, "A new adaptive line enhancer based on singular spectrum analysis," Future Generation Computer Systems, vol. 59, no. 2, pp. 428-434, 2012.

[13] S. Sanei and T. K. M. Lee, "Informed cooperative adaptive line enhancer for detection of spindles from sleep EEG," in Digital Signal Processings DSP 2018, 2018, vol. 1, pp. 1-4.

[14] R. Stockwell, L. Mansinha, and R. Lowe, "Localization of the complex spectrum: the s-transform,"," IEEE Transactions on Signal Processing, vol. 44, no. 4, pp. 998-1001, 1996.

[15] K. J. Blinowska, "Review of the methods of determination of directed connectivity from multichannel data," Medical and Biological Engineering and Computing, vol. 49, no. 5, pp. 521-529, 2011.

[16] S. Monajemi, K. Eftaxias, S. Sanei, and S. H. Ong, "An informed multitask diffusion adaptation approach to study tremor in parkinson's disease," Journal of Selected Topics in Signal Processing, vol. 10, no. 7, pp. 1306-1314, 2016.

[17] K. Eftaxias and S. Sanei, "Discrimination of task-related EEG signals using diffusion adaptation and s-transform coherency," in Machine Learning for Signal Processing, MLSP 2014. IEEE, 2014, vol. 1, pp. 1-6.

[18] S. Sanei, D. Jarchi, and A. Constantinides, Body Sensor Networking, Design and Algorithms, John Wiley \& Sons, 2020. 